\title{
Dynamics of the zeros of analytic continued polynomials and differential equations associated with q-tangent poly- nomials
}

\author{
Cheon Seoung Ryoo ${ }^{\mathrm{a}}$, Kyung Won Hwang ${ }^{\mathrm{b}, *}$, Do Jin Kim ${ }^{\mathrm{c}}$, Nam Soon Jung ${ }^{\mathrm{d}}$ \\ ${ }^{a}$ Department of Mathematics, Hannam University, Daejeon 306-791, Republic of Korea. \\ ${ }^{b}$ Department of Mathematics, Dong-A University, Busan 604-714, Republic of Korea. \\ ${ }^{c}$ Department of Mathematics, Kyungpook National University, Daegu, 702-701, Republic of Korea. \\ ${ }^{d}$ College of Talmage Liberal Arts, Hannam University, Daejeon 306-791, Republic of Korea.
}

Communicated by M. Bohner

\begin{abstract}
In this paper, we study the analytic continuation $T_{q}(s)$ and $T_{q}(s, w)$ of the $q$-Tangent numbers $T_{n, q}$ and $q$-Tangent polynomials $T_{n, q}(x)$ introduced by authors. The new concept of dynamics of the zeros of analytic continued q-tangent polynomials is investigated observing an interesting phenomenon of 'scattering' of the zeros of $T_{q}(s, w)$. Finally, we study linear differential equations arising from the generating functions of q-tangent polynomials giving explicit identities for the q-tangent polynomials.

Keywords: Tangent numbers and polynomials, q-tangent polynomial, q-tangent Zeta function, analytic continuation, analytic continued q-tangent polynomials, zeros, differential equations.
\end{abstract}

2010 MSC: 11B68, 11S40.

(C)2018 All rights reserved.

\section{Introduction}

The computing process is one of the most important research areas nowadays such as solving many mathematical problems with the aid of computers. Using software algorithm, researchers can explore theoretical concepts and numerical experiments much more easily than in the past. There have been lots of research by mathematician in different kinds of the Tangent, Euler, Bernoulli, and Genocchi numbers and polynomials (see [1-17]). Numerical developments as well as experiments of Bernoulli polynomials, Euler polynomials, Genocchi polynomials, and Tangent polynomials have been studied with the significant progress in mathematics and computer science as one of the interesting subjects for the development of computer algorithms. Throughout this paper, we use the following notations: $\mathbb{N}, \mathbb{N}_{0}, \mathbb{R}$, and $\mathbb{C}$ denote the sets of natural numbers, nonnegative integers, real numbers, and complex numbers, respectively. First,

\footnotetext{
*Corresponding author

Email addresses: ryoocs@hnu.kr (Cheon Seoung Ryoo), khwang@dau.ac.kr (Kyung Won Hwang), kimdojin@knu.ac.kr (Do Jin Kim), soonjn@gmail . com (Nam Soon Jung)
}

doi: $10.22436 /$ jnsa.011.06.06

Received: 2017-06-28 Revised: 2018-01-16 Accepted: 2018-03-08 
we consider one of the well known nonlinear differential equations of the first order: Bernoulli equation which is defined as

$$
\frac{d y}{d t}+p(x) y=g(x) y^{m} \quad(m: \text { any real number })
$$

where $p(x)$ and $g(x)$ are continuous functions. For $m=0$ and $m=1$ the equation is linear, and otherwise it is nonlinear. Especially, when $m=2$, the solution of the Bernoulli equation is the form of the exponential function type generating function of the Euler numbers.

We now give the definitions of the $q$ - tangent numbers and polynomials and note that the definition of $q$ - tangent numbers $T_{n, q}$ and polynomials $T_{n, q}(x)$ is introduced in [11] for detail. Let $q$ be a complex number with $|q|<1$. The q-tangent numbers $T_{n, q}$ and polynomials $T_{n, q}(x)$ are defined by means of the generating functions:

$$
\frac{2}{q e^{2 t}+1}=\sum_{n=0}^{\infty} T_{n, q} \frac{t^{n}}{n !}, \quad\left(\frac{2}{q e^{2 t}+1}\right) e^{x t}=\sum_{n=0}^{\infty} T_{n, q}(x) \frac{t^{n}}{n !} .
$$

For $k \in \mathbb{N}$, higher-order tangent numbers $T_{n, q}^{(k)}$ and polynomials, $T_{n, q}^{(k)}(x)(n \geqslant 0)$, were introduced by Ryoo (see [17]). The q-tangent polynomials of higher order, $T_{n, q}^{(k)}(x)$ are defined by means of the following generating function

$$
\left(\frac{2}{q e^{2 t}+1}\right)^{k} e^{x t}=\sum_{n=0}^{\infty} T_{n, q}^{(k)}(x) \frac{t^{n}}{n !} .
$$

The $\mathrm{q}$ - tangent numbers of higher order, $\mathrm{T}_{\mathrm{n}, \mathrm{q}}^{(\mathrm{k})}$ are defined by the following generating function

$$
\left(\frac{2}{q e^{2 t}+1}\right)^{k}=\sum_{n=0}^{\infty} T_{n, q}^{(k)} \frac{t^{n}}{n !}, \quad|2 t+\log q|<\pi .
$$

When $k=1,(1.2)$ and (1.3) will become the corresponding definitions of the q-tangent polynomials $T_{n, q}(x)$ and the $q$-tangent numbers $T_{n, q}$ (see [11]). One can observe that if $q \rightarrow 1$, then $T_{n, q}(x)=T_{n}(x)$ and $\mathrm{T}_{\mathrm{n}, \mathrm{q}}=\mathrm{T}_{\mathrm{n}}$ (see [10]).

As is well known that when $m=2$ a special Bernoulli equation

$$
\frac{d y}{d t}+2 y=y^{2}
$$

has the solution

$$
y=\frac{2}{q e^{2 t}+1}=\sum_{n=0}^{\infty} T_{n, q} \frac{t^{n}}{n !} .
$$

That is, the Bernoulli equation has the solution which is the function of exponential generating function of the q-tangent numbers. Thus, the analytic continued polynomials $T_{q}(s, w)$ is very interesting realistic research area by using computer. It is the purpose of this paper to investigate an interesting phenomenon of 'scattering' from the zeros of the continued q-tangent polynomials $T_{q}(s, w)$ in complex plane.

Theorem 1.1 ([11]). For $n \in \mathbb{N}_{0}$, it satisfies that

$$
\mathrm{T}_{\mathrm{n}, \mathrm{q}}(\mathrm{x})=\sum_{\mathrm{l}=0}^{\mathrm{n}}\left(\begin{array}{c}
\mathrm{n} \\
l
\end{array}\right) \mathrm{T}_{\mathrm{l}, \mathrm{q}} \mathrm{x}^{\mathrm{n}-\mathrm{l}}
$$

From the fact

$$
\frac{\partial}{\partial x} F_{q}(t, x)=t F_{q}(t, x)=\sum_{n=0}^{\infty} \frac{d}{d x} T_{n, q}(x) \frac{t^{n}}{n !},
$$

one can have the important relation

$$
\frac{d}{d x} T_{n, q}(x)=n T_{n-1, q}(x) .
$$


The following integral formula also can be obtained

$$
\int_{0}^{x} T_{n-1, q}(x) d x=\frac{1}{n}\left(T_{n, q}(x)-T_{n, q}\right) .
$$

Then, it is easily deduced that $T_{n, q}(x)$ are polynomials of degree $n$. For example, the list of the first q-tangent's polynomials are as follows:

$$
\begin{aligned}
& T_{0, q}(x)=\frac{2}{1+q}, \\
& T_{1, q}(x)=\frac{-4 q+2 x+2 q x}{(1+q)^{2}}, \\
& T_{2, q}(x)=\frac{-8 q+8 q^{2}-8 q x-8 q^{2} x+2 x^{2}+4 q x^{2}+2 q^{2} x^{2}}{(1+q)^{3}}, \\
& T_{3, q}(x)=\frac{-16 q+64 q^{2}-16 q^{3}-24 q x+24 q^{3} x-12 q x^{2}-24 q^{2} x^{2}-12 q^{3} x^{2}}{(1+q)^{4}}+\frac{2 x^{3}+6 q x^{3}+6 q^{2} x^{3}+2 q^{3} x^{3}}{(1+q)^{4}} .
\end{aligned}
$$

\section{Generating tangent polynomials and numbers}

In [11], the q-tangent zeta function and Hurwitz q-tangent zeta function are introduced by authors. By $q$-tangent zeta function, we consider the function $T_{q}(s)$ as the analytic continuation of q-tangent numbers in this section.

Definition 2.1. For $s \in \mathbb{C}$ with $\operatorname{Re}(s)>0$, we define the q-tangent zeta function by

$$
\zeta_{\mathrm{T}, \mathrm{q}}(\mathrm{s})=2 \sum_{\mathrm{n}=1}^{\infty} \frac{(-1)^{\mathrm{n}} \mathrm{q}^{\mathrm{n}}}{(2 \mathrm{n})^{\mathrm{s}}} \text {, see [11]. }
$$

Notice that the q-tangent zeta function can be analytically continued to the whole complex plane, and these zeta function have the values of the q-tangent numbers at negative integers. That is, $\mathrm{q}$-tangent numbers are related to the q-tangent zeta function as

$$
\zeta_{\mathrm{T}, \mathrm{q}}(-\mathrm{n})=\mathrm{T}_{\mathrm{n}, \mathrm{q}}
$$

Definition 2.2. We define the Hurwitz q-tangent zeta function $\zeta_{T, q}(s, x)$ for $s \in \mathbb{C}$ with $\operatorname{Re}(s)>0$ by

$$
\zeta_{T, q}(s, x)=2 \sum_{n=0}^{\infty} \frac{(-1)^{n} q^{n}}{(2 n+x)^{s}} \text {, see [11]. }
$$

q-Tangent polynomials are related to the Hurwitz q-tangent zeta function as

$$
\zeta_{\mathrm{T}, \mathrm{q}}(-\mathrm{n}, \mathrm{x})=\mathrm{T}_{\mathrm{n}, \mathrm{q}}(\mathrm{x}) \text {. }
$$

We now consider the function $T_{q}(s)$ as the analytic continuation of q-tangent numbers. From the above analytic continuation of q-tangent numbers, we consider

$$
\mathrm{T}_{\mathrm{n}, \mathrm{q}} \mapsto \mathrm{T}_{\mathrm{q}}(\mathrm{s}), \quad \zeta_{\mathrm{T}, \mathrm{q}}(-\mathrm{n})=\mathrm{T}_{\mathrm{n}, \mathrm{q}} \mapsto \zeta_{\mathrm{T}, \mathrm{q}}(-\mathrm{s})=\mathrm{T}_{\mathrm{q}}(\mathrm{s}) .
$$

All the q-tangent numbers $T_{n, q}$ agree with $T_{q}(n)$, the analytic continuation of $q$-tangent numbers evaluated at $n$ (see Figure 1),

$$
T_{n, q}=T_{q}(n) \text { for } n \geqslant 1, \quad \text { except } T_{q}(0)=\frac{-2 q}{1+q} \text {, but } T_{0, q}=\frac{2}{1+q} \text {. }
$$




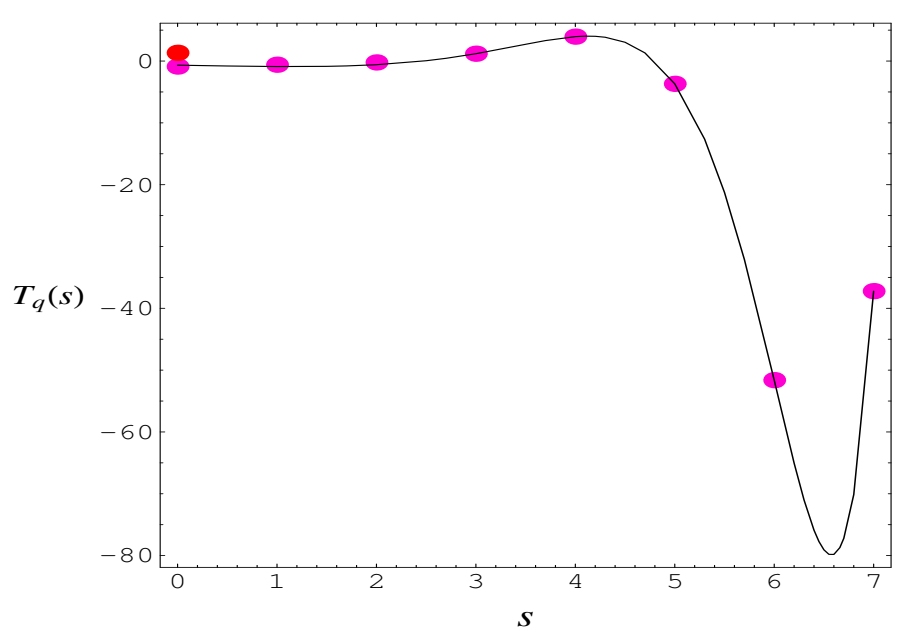

Figure 1: The curve $T_{q}(s)$ runs through the points of all $T_{n, q}$ except $T_{0, q}$.

In Figure 1, we choose $q=\frac{1}{2}$. In fact, we can express $T_{q}^{\prime}(s)$ in terms of $\zeta_{T, q}^{\prime}(s)$, the derivative of $\zeta_{T}(s)$.

$$
\mathrm{T}_{\mathrm{q}}(\mathrm{s})=\zeta_{\mathrm{T}, \mathrm{q}}(-\mathrm{s}), \quad \mathrm{T}_{\mathrm{q}}^{\prime}(\mathrm{s})=-\zeta_{\mathrm{T}, \mathrm{q}}^{\prime}(-\mathrm{s}), \quad \mathrm{T}_{\mathrm{q}}^{\prime}(2 \mathrm{n}+1)=-\zeta_{\mathrm{T}, \mathrm{q}}^{\prime}(-2 \mathrm{n}-1) \text { for } \mathrm{n} \in \mathbb{N}_{0} .
$$

From the relation (2.3), we can define the other analytic continued half of q-tangent numbers

$$
\mathrm{T}_{\mathrm{q}}(\mathrm{s})=\zeta_{\mathrm{T}, \mathrm{q}}(-\mathrm{s}), \quad \mathrm{T}_{\mathrm{q}}(-\mathrm{s})=\zeta_{\mathrm{T}, \mathrm{q}}(\mathrm{s}) \Rightarrow \mathrm{T}_{\mathrm{q}}(-\mathrm{n})=\zeta_{\mathrm{T}, \mathrm{q}}(\mathrm{n}), \mathrm{n} \in \mathbb{N} .
$$

By (2.4), we obtain

$$
\lim _{n \rightarrow \infty} T_{-n, q}=\zeta_{T, q}(n)=0 .
$$

The curve $\mathrm{T}_{\mathrm{q}}(\mathrm{s})$ runs through the points $\mathrm{T}_{-n, \mathrm{q}}=\mathrm{T}_{\mathrm{q}}(-n)$ and grows $\sim 0$ asymptotically as $-n \rightarrow \infty$ (see Figure 2).

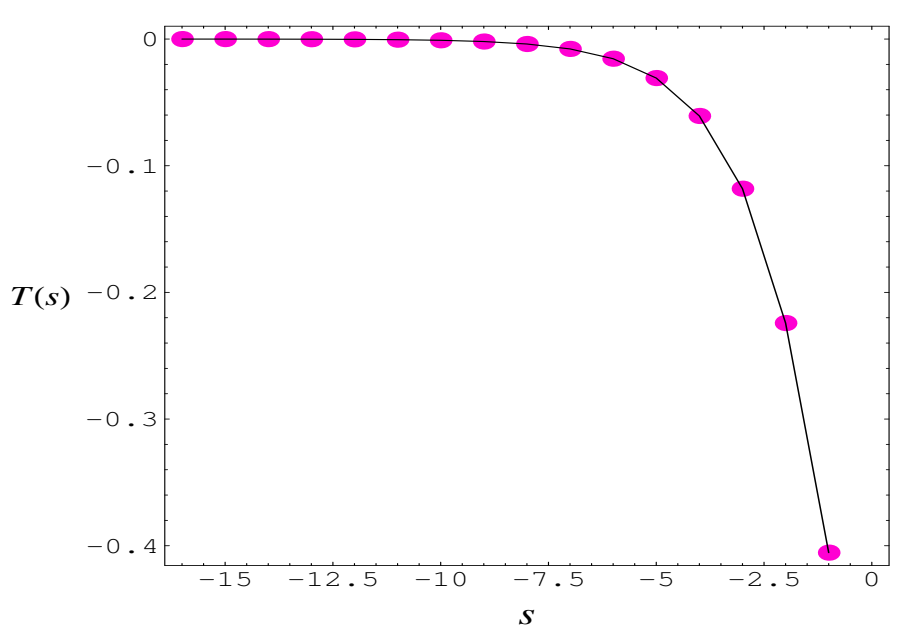

Figure 2: The curve $T_{q}(s)$ runs through the points $T_{-n, q}$ for $q=\frac{1}{2}$.

\section{Analytic continuation of q-tangent polynomials $T_{n, q}(x)$}

In this section, we investigate the analytic continued q-tangent polynomials. Looking back at (2.1) and (2.4), for consistency with the definition of $T_{n, q}(x)=T_{q}(n, x)$, q-tangent polynomials should be 
analogously redefined as

$$
T_{q}(0, x)=-q T_{0, q}(x), \quad T_{q}(n, x)=\sum_{l=0}^{n}\left(\begin{array}{l}
n \\
l
\end{array}\right) T_{l, q} x^{n-l} .
$$

Let $\Gamma(s)$ be the gamma function. Then one can obtain the analytic continuation as follows:

$$
\begin{aligned}
& \mathrm{n} \mapsto \mathrm{s} \in \mathbb{R}, \mathrm{x} \mapsto w \in \mathbb{C}, \\
& \mathrm{T}_{0, \mathrm{q}} \mapsto \mathrm{T}_{\mathrm{q}}(0)=-\frac{1}{\mathrm{q}} \zeta_{\mathrm{T}, \mathrm{q}}(0), \\
& \mathrm{T}_{\mathrm{k}, \mathrm{q}} \mapsto \mathrm{T}_{\mathrm{q}}(\mathrm{k}+\mathrm{s}-[\mathrm{s}])=\zeta_{\mathrm{T}, \mathrm{q}}(-(\mathrm{k}+(\mathrm{s}-[\mathrm{s}]))), \\
& \left(\begin{array}{l}
n \\
k
\end{array}\right) \mapsto \frac{\Gamma(1+s)}{\Gamma(1+k+(s-[s])) \Gamma(1+[s]-k)} \\
& \Rightarrow \mathrm{T}_{\mathrm{n}, \mathrm{q}}(w) \mapsto \mathrm{T}_{\mathrm{q}}(\mathrm{s}, w) \\
& =\sum_{k=-1}^{[s]} \frac{\Gamma(1+s) T_{q}(k+s-[s]) w^{[s]-k}}{\Gamma(1+k+(s-[s])) \Gamma(1+[s]-k)} \\
& =\sum_{k=0}^{[s]+1} \frac{\left(\Gamma(1+s) T_{q}((k-1)+s-[s]) w^{[s]+1-k}\right.}{\Gamma(k+(s-[s])) \Gamma(2+[s]-k)},
\end{aligned}
$$

where $[s]$ denotes the integer part of $s$, and so $s-[s]$ gives the fractional part.

By (3.1), we have analytic continuation of $q$-tangent polynomials for $q=\frac{1}{2}$.

$$
\begin{aligned}
\mathrm{T}_{\mathrm{q}}(0, w) & \approx 1.33333, \\
\mathrm{~T}_{\mathrm{q}}(1, w) & \approx-0.888889+1.33333 w, \\
\mathrm{~T}_{\mathrm{q}}(1.1, w) & \approx-0.895303-0.764176 w-0.023564 w^{2}, \\
\mathrm{~T}_{\mathrm{q}}(1.3, w) & \approx-0.891003-0.974331 w-0.093005 w^{2}, \\
\mathrm{~T}_{\mathrm{q}}(1.5, w) & \approx-0.857828-1.200721 w-0.198212 w^{2}, \\
\mathrm{~T}_{\mathrm{q}}(1.7, w) & \approx-0.787587-1.435791 w-0.346716 w^{2}, \\
\mathrm{~T}_{\mathrm{q}}(1.9, w) & \approx-0.671156-1.667882 w-0.545938 w^{2}, \\
\mathrm{~T}_{\mathrm{q}}(2, w) & \approx-0.592592-1.777777 w+1.333333 w^{2} .
\end{aligned}
$$

By using (3.2), we plot the deformation of the curve $T_{q}(1, w)$ into the curve of $T_{q}(2, w)$ via the real analytic continuation $T_{q}(s, w), 1 \leqslant s \leqslant 2, w \in \mathbb{R}$ (see Figure 3).

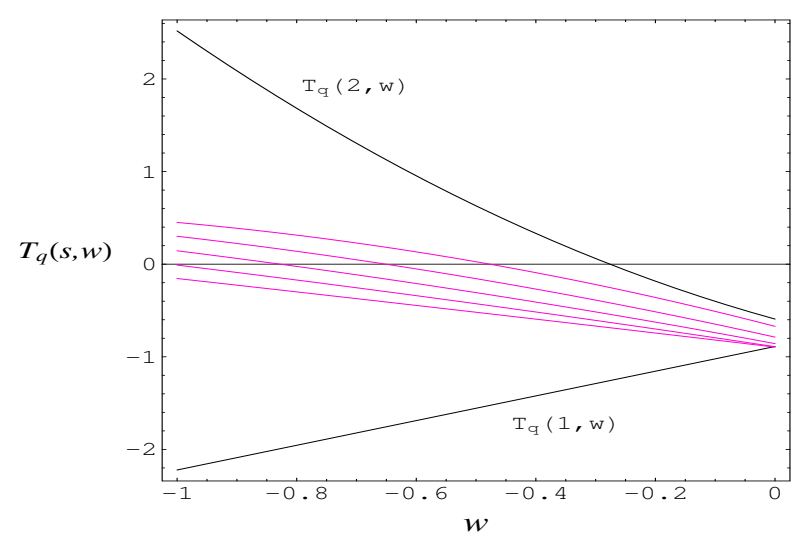

Figure 3: The curve of $T_{\mathrm{q}}(s, w), 1 \leqslant s \leqslant 2,-0.6 \leqslant w \leqslant 0$. 
In [14], we observe that $T_{\mathbf{q}}(n, w), w \in \mathbb{C}$, has $\operatorname{Im}(w)=0$ reflection symmetry analytic complex functions (Figure 4). The obvious corollary is that the zeros of $T_{q}(n, w)$ will also inherit these symmetries.

$$
\text { If } T_{q}\left(n, w_{0}\right)=0 \text {, then } T_{q}\left(n, w_{0}^{*}\right)=0,
$$

where $*$ denotes complex conjugation.

Next, we investigate the beautiful zeros of the $T_{q}(s, w)$ by using a computer. We plot the zeros of $\mathrm{T}_{\mathrm{q}}(\mathrm{s}, w)$ for $\mathrm{s}=10,10.6,10.8,11, \mathrm{q}=\frac{1}{2}$, and $w \in \mathbb{C}$ (Figure 4$)$.
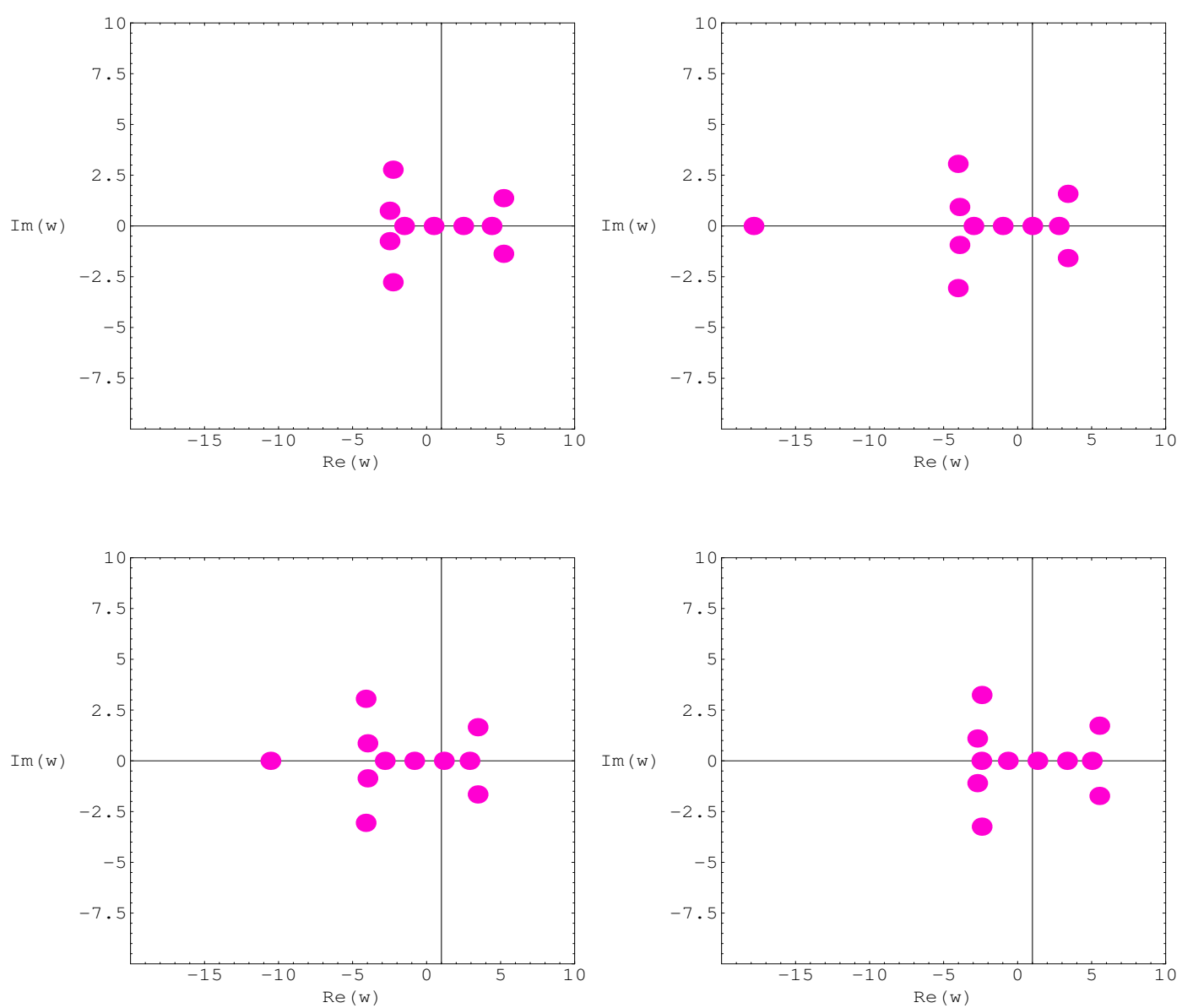

Figure 4: $\mathrm{T}_{\mathrm{q}}(\mathrm{s}, \mathrm{w})$ for $\mathrm{s}=10,10.6,10.8,11$.

In Figure 4 (top-left), we choose $s=10$. In Figure 4 (top-right), we choose $s=10.6$. In Figure 4 (bottom-left), we choose $s=10.8$. In Figure 4 (bottom-right), we choose $s=11$.

Since

$$
\sum_{n=0}^{\infty} T_{n, q^{-1}}(2-x) \frac{(-1)^{n} t^{n}}{n !}=\frac{2}{q^{-1} e^{-2 t}+1} e^{(2-x)(-t)}=\frac{2 q}{q e^{2 t}+1} e^{x t}=\sum_{n=0}^{\infty} q T_{n, q}(x) \frac{t^{n}}{n !},
$$

we obtain

$$
\mathrm{qT}_{\mathrm{n}, \mathrm{q}}(\mathrm{x})=(-1)^{\mathrm{n}} \mathrm{T}_{\mathrm{n}, \mathrm{q}^{-1}}(2-\mathrm{x}) \text {. }
$$

The question is: what happens with the reflexive symmetry (3.3), when one considers q-tangent polynomials? Prove that $T_{q}(n, w), w \in \mathbb{C}$, has not $\operatorname{Re}(w)=1$ reflection symmetry analytic complex functions (Figure 4). However, we observe that $\mathrm{T}_{\mathrm{q}}(\mathrm{s}, w), w \in \mathbb{C}$, has $\operatorname{Im}(x)=0$ reflection symmetry analytic complex functions (Figure 4). 
Stacks of zeros of $T_{q}(s, w)$ for $s=n+1 / 2,1 \leqslant n \leqslant 30$ form a 3-D structure are presented (Figure 5).
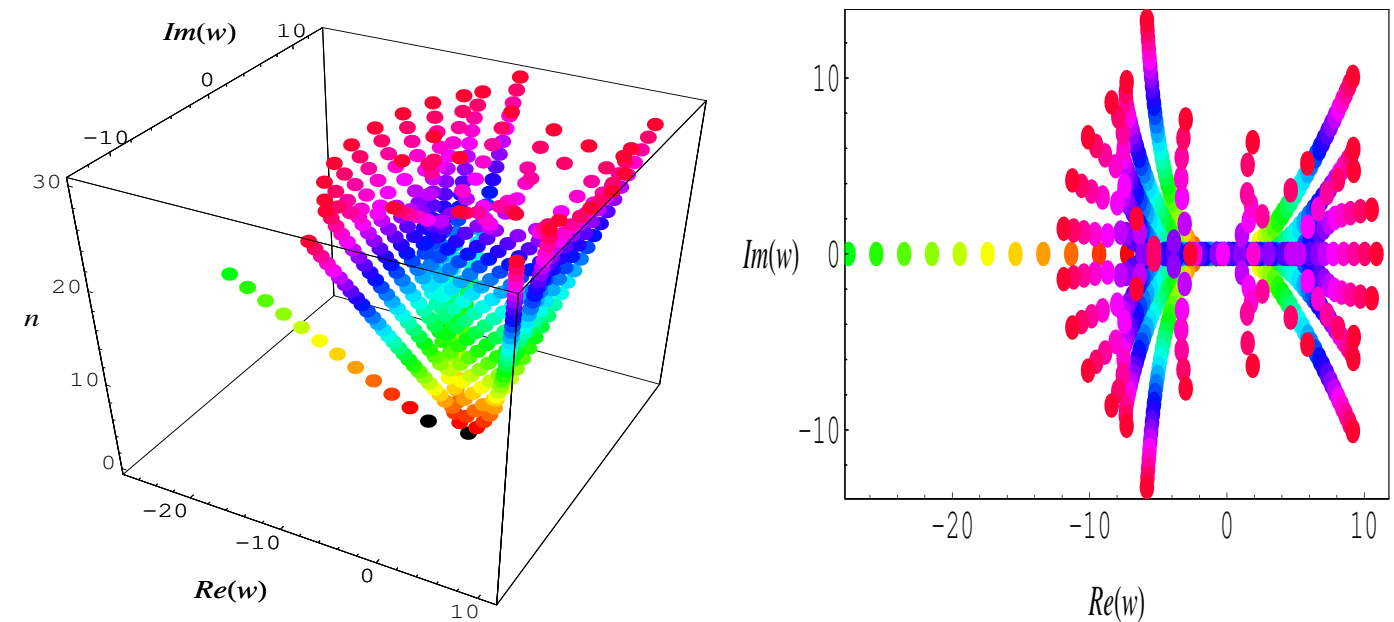

$\operatorname{Re}(w)$
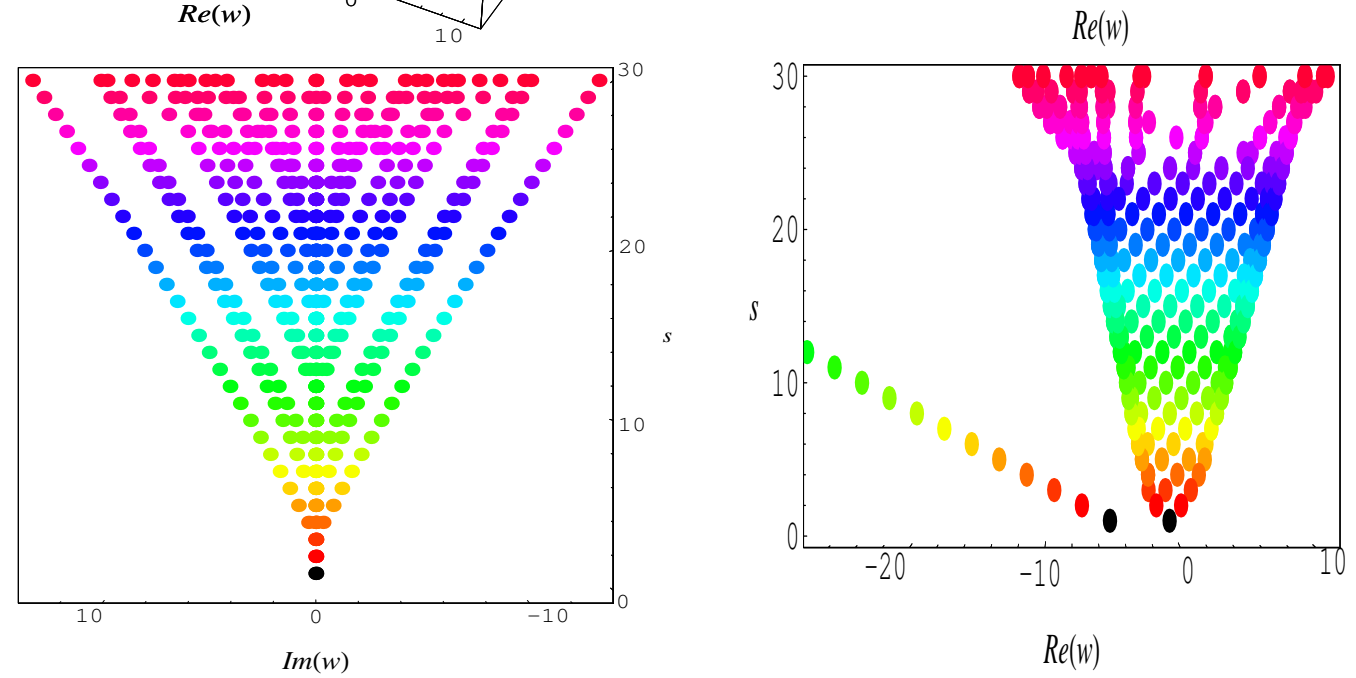

Figure 5: Stacks of zeros of $T_{q}(s, w)$ for $1 \leqslant n \leqslant 30$.

Our numerical results for approximate solutions of real zeros of $\mathrm{T}_{\mathrm{q}}(\mathrm{s}, w), \mathrm{q}=\frac{1}{2}$ are displayed. We observe a remarkably regular structure of the complex roots of q-tangent polynomials. We hope to verify a remarkably regular structure of the complex roots of q-tangent polynomials (Table 1).

Table 1: Numbers of real and complex zeros of $\mathrm{T}_{\mathrm{q}}(\mathrm{s}, w)$.

\begin{tabular}{ccc}
\hline s & real zeros & complex zeros \\
\hline \hline 1.5 & 2 & 0 \\
2.5 & 3 & 0 \\
3.5 & 4 & 0 \\
4.5 & 3 & 2 \\
5.5 & 4 & 2 \\
6.5 & 5 & 2 \\
7.5 & 4 & 4 \\
8.5 & 5 & 4 \\
9.5 & 4 & 6 \\
10 & 4 & 6 \\
10.6 & 5 & 6 \\
10.8 & 5 & 6 \\
11 & 5 & 6 \\
\hline
\end{tabular}


Next, we calculated an approximate solution satisfying $T_{q}(s, w), q=\frac{1}{2}, w \in \mathbb{R}$. The results are given in Table 2.

Table 2: Approximate solutions of $\mathrm{T}(\mathrm{s}, w)=0, w \in \mathbb{R}$.

\begin{tabular}{llllll}
\hline $\mathrm{s}$ & $\mathrm{W}$ & \multicolumn{5}{c}{} \\
\hline \hline 6 & -0.943798, & 1.03202, & 3.05571, & 3.91507 & \\
6.5 & -15.4379, & -2.44149, & -0.536778, & 1.62862, & 1.89524 \\
7 & -1.68478, & -0.105853, & 1.89415 & & \\
7.5 & -17.4652, & -3.10753, & -1.67601, & 0.32492 & \\
8 & -2.00343, & -1.25508, & 0.755751, & 2.75566 & \\
8.5 & -19.492, & -3.25996, & -2.93625, & -0.813354, & 1.18527 \\
9 & -0.382455, & 1.61755, & 3.60912 & & \\
9.5 & -21.5185, & -1.95122, & 0.0484235, & 2.01619 & \\
10 & -1.51713, & 0.479293, & 2.47929, & 4.39156 & \\
10.6 & -17.836, & -2.97915, & -1.00366, & 0.99635, & 2.78504 \\
10.8 & -10.5456, & -2.82217, & -0.831306, & 1.16872, & 2.90219 \\
11 & -2.44574, & -0.658954, & 1.34105, & 3.34112, & 5.01339 \\
\hline
\end{tabular}

In Figure 5, we plot the real zeros of the q-tangent polynomials $T_{q}(s, w)$ for $s=n+1 / 2, q=1 / 2, w \in \mathbb{C}$ (Figure 6).

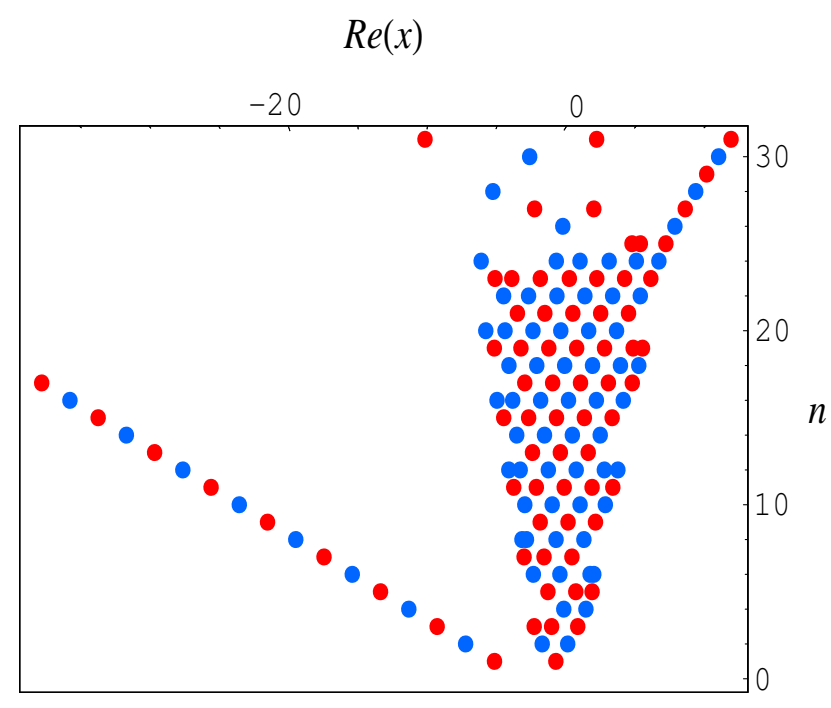

Figure 6: Real zeros of $\mathrm{T}_{\mathrm{q}}(\mathrm{s}, w)$.

The q-tangent polynomials $T_{q}(n, w)$ are of degree $n$. Thus, $T_{q}(n, w)$ has $n$ zeros and $T_{q}(n+1, w)$ has $n+1$ zeros. When discrete $n$ is analytic continued to continuous parameter $s$, it naturally provides the following question:

How does $T_{q}(s, w)$, the analytic continuation of $T_{q}(n, w)$, pick up an additional zero as $s$ increases continuously by one?

This introduces the exciting concept of the dynamics of the zeros of analytic continued q-tangent polynomials, the idea of looking at how the zeros move about in the $w$ complex plane as we vary the parameter s.

To have a physical picture of the motion of the zeros in the complex $w$ plane, imagine that each time as $s$ increases gradually and continuously by one, an additional real zero flies in from positive infinity along the real positive axis, gradually slowing down as if "it is flying through a viscous medium". 
For more studies and results in this subject you may see [7, 12-15].

\section{Differential equations associated with q-tangent polynomials}

Differential equations arising from the generating functions of special polynomials are studied by many authors in order to give explicit identities for special polynomials (see $[6,14])$. In this section, we study linear differential equations arising from the generating functions of q-tangent polynomials. We give explicit identities for the q-tangent polynomials. In this section, we study linear differential equations arising from the generating functions of q-tangent polynomials. Let

$$
H=H(t, q)=\frac{2}{q e^{2 t}+1}, \quad F=F(t, q, x)=\left(\frac{2}{q e^{2 t}+1}\right) e^{x t}
$$

Then, by (4.1), we get

$$
H^{(1)}=\frac{d}{d t} H(t, q)=\frac{d}{d t}\left(\frac{2}{q e^{2 t}+1}\right)=-q\left(\frac{2}{q e^{2 t}+1}\right)^{2} e^{2 t}
$$

Hence we have

$$
\mathrm{H}^{(1)}=-\mathrm{q} \mathrm{H}^{2} e^{2 \mathrm{t}}
$$

By (4.1), we have

$$
\begin{aligned}
& F^{(1)}=\frac{d}{d t} F(t, q, x)=\frac{d}{d t}\left(\frac{2}{q e^{2 t}+1}\right) e^{x t} \\
& =-q\left(\frac{2}{q e^{2 t}+1}\right)^{2} e^{(x+2) t}+x\left(\frac{2}{q e^{2 t}+1}\right) e^{x t}=\left(-q H e^{2 t}+x\right) F(t, q, x), \\
& F^{(2)}=\left(\frac{d}{d t}\right)^{2} F(t, q, x)=\left(-1 q H^{(1)} e^{2 t}-2 x q H e^{2 t}\right) F+\left(-q H e^{2 t}+x\right) F^{(1)} \\
& =(-1)^{2} 2 q^{2} H^{2} e^{4 t} F+(-1) 2 q x H e^{2 t} F+(-1) 2 q H e^{2 t} F+x^{2} F \\
& =\left((-1)^{2} 2 q^{2} H^{2} e^{4 t}+(-1)(2 q x+2 q) H e^{2 t}+x^{2}\right) F(t, q, x) \text {, }
\end{aligned}
$$

and

$$
\begin{aligned}
F^{(3)}= & \left(\frac{d}{d t}\right)^{3} F(t, q, x) \\
= & (-1)^{2} 4 q^{2} H H^{(1)} e^{4 t} F+(-1)^{2} 8 q^{2} H^{2} e^{4 t} F+(-1)^{2} 2 q^{2} H^{2} e^{4 t} F^{(1)} \\
& +(-2)(q x+q) H^{(1)} e^{2 t} F+(-4)(q x+q) H e^{2 t} F+(-2)(q x+q) H e^{2 t} F^{(1)} \\
= & (-1)^{3} 6 q^{3} H^{3} e^{6 t} F(t, q, x)+(-1)^{2}\left(8 q^{2}+2 q^{2} x+2 q^{2} x+2 q^{2}\right) H^{2} e^{4 t} F(t, q, x) \\
& +(-1)\left(4 q x+4 q+q x^{2}\right) H e^{2 t} F(t, q, x)+x^{3} F(t, q, x) .
\end{aligned}
$$

Continuing this process, we can guess that

$$
F^{(N)}=\left(\frac{d}{d t}\right)^{N} F(t, q, x)=\left(\sum_{i=0}^{N}(-1)^{i} a_{i}(N, q, x) H^{i} e^{2 i t}\right) F(t, q, x), \quad(N=0,1,2, \ldots) .
$$

Taking the derivative with respect to $t$ in (2.2), we obtain

$$
F^{(N+1)}=\frac{d F^{(N)}}{d t}=\left(\sum_{i=0}^{N}(-1)^{i} i a_{i}(N, q, x) H^{i-1} H^{(1)} e^{2 i t}+\sum_{i=0}^{N}(-1)^{i} 2 i a_{i}(N, q, x) H^{i} e^{2 i t}\right) F(t, q, x)
$$




$$
\begin{aligned}
& +\left(\sum_{i=0}^{N}(-1)^{i} a_{i}(N, q, x) H^{i} e^{2 i t}\right) F^{(1)}(t, q, x) \\
= & \left(\sum_{i=0}^{N}(-1)^{i+1} q(i+1) a_{i}(N, q, x) H^{i+1} e^{2(i+1) t}\right) F(t, q, x) \\
& +\left(\sum_{i=0}^{N}(-1)^{i}(2 i+x) a_{i}(N, q, x) H^{i} e^{2 i t}\right) F(t, q, x) \\
= & \left(\sum_{i=0}^{N}(-1)^{i}(2 i+x) a_{i}(N, q, x) H^{i} e^{2 i t}\right) F(t, q, x) \\
& +\left(\sum_{i=1}^{N+1}(-1)^{i} q i a_{i-1}(N, q, x) H^{i} e^{2 i t}\right) F(t, q, x) .
\end{aligned}
$$

On the other hand, by replacing $N$ by $N+1$ in (4.3), we get

$$
F^{(N+1)}=\left(\sum_{i=0}^{N+1}(-1)^{i} a_{i}(N+1, q, x) H^{i} e^{2 i t}\right) F(t, q, x) .
$$

By (4.4) and (4.5), we have

$$
\begin{gathered}
\left(\sum_{i=0}^{N}(x+2 i) a_{i}(N, q, x) H^{i} e^{2 i t}+\sum_{i=1}^{N+1}(-1)^{i} q i a_{i-1}(N, q, x) H^{i} e^{2 i t}\right) F(t, q, x) \\
=\left(\sum_{i=0}^{N+1}(-1)^{i} a_{i}(N+1, q, x) H^{i} e^{2 i t}\right) F(t, q, x) .
\end{gathered}
$$

Comparing the coefficients on both sides of (4.6), we obtain

$$
a_{0}(N+1, q, x)=x a_{0}(N, q, x), \quad a_{N+1}(N+1, q, x)=q(N+1) a_{N}(N, q, x),
$$

and

$$
a_{i}(N+1, q, x)=(x+2 i) a_{i}(N, q, x)+q i a_{i-1}(N, q, x),(1 \leqslant i \leqslant N) .
$$

In addition, by (4.2) and (4.3), we get

$$
F=F^{(0)}=a_{0}(0, q, x) F(t, q, x)=F(t, q, x) .
$$

Thus, by (4.9), we obtain

$$
a_{0}(0, q, x)=1
$$

It is not difficult to show that

$$
\begin{aligned}
-q H e^{2 t} F(t, q, x)+x F(t, q, x) & =\sum_{i=0}^{1}(-1)^{i} a_{i}(1, q, x) H^{i} e^{2 i t} F(t, q, x) \\
& =a_{0}(1, q, x) F(t, q, x)+(-1) a_{1}(1, q, x) H e^{2 t} F(t, q, x) .
\end{aligned}
$$

Thus, by (4.10), we also get

$$
a_{0}(1, q, x)=x, \quad a_{1}(1, q, x)=q
$$

From (4.7), we note that

$$
a_{0}(N+1, q, x)=x a_{0}(N, q, x)=x^{2} a_{0}(N-1, q, x)=\cdots=x^{N+1},
$$


and

$$
a_{N}(N+1, q, x)=q(N+1) a_{N}(N, q, x)=\cdots=q^{(N+1)}(N+1) !
$$

For $i=1,2,3$ in $(4.8)$, we get

$$
\begin{aligned}
& a_{1}(N+1, q, x)=q \sum_{k=0}^{N}(x+2)^{k} a_{0}(N-k, q, x), \\
& a_{2}(N+1, q, x)=2 q \sum_{k=0}^{N-1}(x+4)^{k} a_{1}(N-k, q, x), \\
& a_{3}(N+1, q, x)=3 q \sum_{k=0}^{N-2}(x+6)^{k} a_{2}(N-k, q, x) .
\end{aligned}
$$

Continuing this process, we can deduce that, for $1 \leqslant i \leqslant N$,

$$
\left.a_{i}(N+1, q, x)\right)=i q \sum_{k=0}^{N-i+1}(x+2 i)^{k} a_{i-1}(N-k, q, x) .
$$

Now, we give explicit expressions for $a_{i}(N+1, q, x)$. By (4.11) and (4.12), we get

$$
\begin{aligned}
a_{1}(N+1, q, x) & =q \sum_{k_{1}=0}^{N}(x+2)^{k_{1}} a_{0}\left(N-k_{1}, q, x\right)=q \sum_{k_{1}=0}^{N}(x+2)^{k_{1}} x^{N-k_{1}}, \\
a_{2}(N+1, q, x) & =2 q \sum_{k_{2}=0}^{N-1}(x+4)^{k_{2}} a_{1}\left(N-k_{2}, q, x\right)=2 q^{2} \sum_{k_{2}=0}^{N-1} \sum_{k_{1}=0}^{N-k_{2}-1}(x+4)^{k_{2}}(x+2)^{k_{1}} \chi^{N-k_{2}-k_{1}-1}, \\
a_{3}(N+1, q, x) & =3 q \sum_{k_{3}=0}^{N-2}(x+6)^{k_{3}} a_{2}\left(N-k_{3}, q, x\right) \\
& =6 q^{3} \sum_{k_{3}=0}^{N-2} \sum_{k_{2}=0}^{N-k_{3}-2} \sum_{k_{1}=0}^{N-k_{3}-k_{2}-2}(x+6)^{k_{3}}(x+4)^{k_{2}}(x+2)^{k_{1}} x^{N-k_{2}-k_{2}-k_{1}-2} .
\end{aligned}
$$

Continuing this process, we have

$$
a_{i}(N+1)=i ! q^{i} \sum_{k_{i}=0}^{N-i+1} \sum_{k_{i-1}=0}^{N-k_{i}-i+1} \cdots \sum_{k_{1}=0}^{N-k_{i}-\cdots-k_{2}-i+1}(x+2 i)^{k_{i}} \cdots(x+2)^{k_{1}} \chi^{N-k_{i}-\cdots-k_{1}-i+1} .
$$

Therefore, by (4.13), we obtain the following theorem.

Theorem 4.1. For $\mathrm{N}=0,1,2, \ldots$, the functional equations

$$
F^{(N)}=\left(\sum_{i=0}^{N}(-1)^{i} a_{i}(N, q, x)\left(\frac{2}{q e^{2 t}+1}\right)^{i} e^{2 i t}\right) F
$$

have a solution

where

$$
F=F(t, q, x)=\left(\frac{2}{q e^{2 t}+1}\right) e^{x t}
$$

$$
\begin{aligned}
a_{0}(N, q, x) & =x^{N} \\
a_{N}(N, q, x) & =N ! q^{N}, \\
a_{i}(N, q, x) & =i ! q^{i} \sum_{k_{i}=0}^{N-i} \sum_{k_{i-1}=0}^{N-k_{i}-i} \cdots \sum_{k_{1}=0}^{N-k_{i}-\cdots-k_{2}-i}(x+2 i)^{k_{i}} \cdots(x+2)^{k_{1}} x^{N-k_{i}-\cdots-k_{1}-i}, \quad(1 \leqslant i \leqslant N) .
\end{aligned}
$$


From (1.1), we note that

$$
F^{(N)}=\left(\frac{d}{d t}\right)^{N} F(t, q, x)=\sum_{k=0}^{\infty} T_{k+N, q}(x) \frac{t^{k}}{k !} .
$$

From Theorem 4.1, (1.3), and (4.14), we can derive the following equation:

$$
\begin{aligned}
\sum_{k=0}^{\infty} T_{k+N, q}(x) \frac{t^{k}}{k !}=F^{(N)} & =\left(\sum_{i=0}^{N}(-1)^{i} a_{i}(N, q, x)\left(\frac{2}{q e^{2 t}+1}\right)^{i} e^{2 i t}\right) F \\
& =\sum_{i=0}^{N}(-1)^{i} a_{i}(N, q, x) e^{(x+2 i) t}\left(\frac{2}{q e^{2 t}+1}\right)^{i+1} \\
& =\sum_{i=0}^{N}(-1)^{i} a_{i}(N, q, x)\left(\sum_{k=0}^{\infty} T_{k, q}^{(i+1))}(x+2 i) \frac{t^{k}}{k !}\right) \\
& =\sum_{k=0}^{\infty}\left(\sum_{i=0}^{N}(-1)^{i} a_{i}(N, q, x) T_{k, q}^{(i+1))}(x+2 i)\right) \frac{t^{k}}{k !} .
\end{aligned}
$$

By comparing the coefficients on both sides of (4.15), we obtain the following theorem.

Theorem 4.2. For $\mathrm{k}=0,1, \ldots$, and $\mathrm{N}=0,1,2, \ldots$, we have

$$
\begin{aligned}
\mathrm{T}_{k+N, q}(x) & =\sum_{i=0}^{N}(-1)^{i} a_{i}(N, q, x) T_{k, q}^{(i+1)}(x+2 i) \\
& =\sum_{i=0}^{N} \sum_{l=0}^{k}\left(\begin{array}{l}
k \\
l
\end{array}\right)(-1)^{i}(2 i)^{k-l} a_{i}(N, q, x) T_{l, q}^{(i+1)}(x),
\end{aligned}
$$

where

$$
\begin{aligned}
a_{0}(N, q, x) & =x^{N}, \\
a_{N}(N, q, x) & =N ! q N \\
a_{i}(N, q, x) & =i ! q^{i} \sum_{k_{i}=0}^{N-i} \sum_{k_{i-1}=0}^{N-k_{i}-i} \cdots \sum_{k_{1}=0}^{N-k_{i}-\cdots-k_{2}-i}(x+2 i)^{k_{i}} \cdots(x+2)^{k_{1}} x^{N-k_{i}-\cdots-k_{1}-i}, \quad(1 \leqslant i \leqslant N) .
\end{aligned}
$$

Let us take $k=0$ in (4.16). Then, we have the following corollary.

Corollary 4.3. For $\mathrm{N}=0,1,2, \ldots$, we have

$$
\mathrm{T}_{\mathrm{N}, \mathrm{q}}(\mathrm{x})=\sum_{i=0}^{\mathrm{N}}(-1)^{i} \mathrm{a}_{i}(\mathrm{~N}, \mathrm{q}, x) \mathrm{T}_{0, \mathrm{q}}^{(i+1)}(x+2 i) .
$$

For $\mathrm{N}=1,2, \ldots$, the functional equations

$$
F^{(N)}=\left(\sum_{i=0}^{N}(-1)^{i} a_{i}(N, q, x)\left(\frac{2}{q e^{2 t}+1}\right)^{i} e^{2 i t}\right) F
$$

have a solution

$$
F=F(t, q, x)=\left(\frac{2}{q e^{2 t}+1}\right) e^{x t}
$$

Here is a plot of the surface for this solution. 


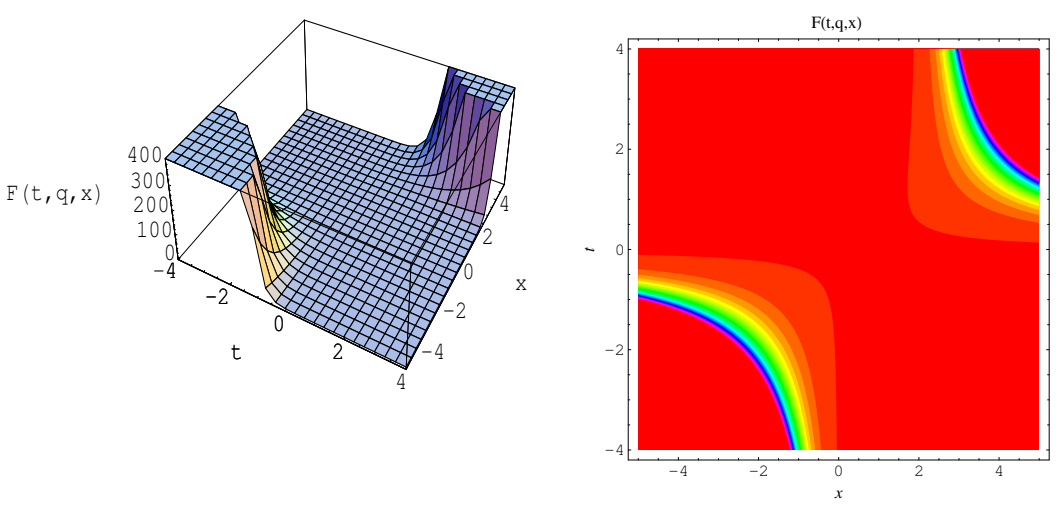

Figure 7: The surface for the solution $F(t, q, x)$.

In Figure 7, we choose $q=\frac{1}{2}$ and plot of the surface for this solution (left). In Figure 4 (right), it shows a higher-resolution density plot of the solution.

\section{Acknowledgment}

This work was supported by the Dong-A university research fund.

\section{References}

[1] R. Ayoub, Euler and the zeta function, Amer. Math. Monthly, 81 (1974), 1067-1086. 1

[2] A. Erdélyi, W. Magnus, F. Oberhettinger, F. G. Tricomi, Higher Transcendental Functions, Vol. III., Krieger Publishing Co., Melbourne, (1981).

[3] J. Y. Kang, H. Y. Lee, N. S. Jung, Some relations of the twisted q-Genocchi numbers and polynomials with weight $\alpha$ and weak Weight $\beta$, Abstr. Appl. Anal., 2012 (2012), 9 pages.

[4] T. Kim, Euler numbers and polynomials associated with zeta functions, Abstr. Appl. Anal., 2008 (2008), 11 pages.

[5] M.-S. Kim, S. Hu, On p-adic Hurwitz-type Euler Zeta functions, J. Number Theory, 132 (2012), 2977-3015.

[6] T. Kim, D. S. Kim, C. S. Ryoo, H. I. Kwon, Differential equations associated with Mahler and Sheffer-Mahler polynomials, submitted for publication. 4

[7] T. Kim, C. S. Ryoo, L. C. Jang, S. H. Rim, Exploring the q-Riemann Zeta function and q-Bernoulli polynomials, Discrete Dyn. Nat. Soc., 2005 (2005), 171-181. 3

[8] H. Ozden, Y. Simsek, A new extension of q-Euler numbers and polynomials related to their interpolation functions, Appl. Math. Lett., 21 (2008), 934-938.

[9] S. H. Rim, K. H. Park, E. J. Moon, On Genocchi numbers and polynomials, Abstr. Appl. Anal., 2008 (2008), 7 pages.

[10] C. S. Ryoo, A Note on the Tangent Numbers and Polynomials, Adv. Studies Theor. Phys., 7 (2013), 447-454. 1

[11] C. S. Ryoo, On the q-Tangent Numbers and Polynomials, Appl. Math. Sci. (Ruse), 7 (2013), 4935-4941. 1, 1, 1.1, 2, 2.1, 2.2

[12] C. S. Ryoo, A Numerical investigation on the zeros of the tangent polynomials, J. Appl. Math. Inform., 32 (2014), 315-322. 3

[13] C. S. Ryoo, Analytic Continuation of Euler Polynomials and the Euler Zeta Function, Discrete Dyn. Nat. Soc., 2014 (2014), 6 pages.

[14] C. S. Ryoo, Differential equations associated with tangent numbers, J. Appl. Math. Inform., 34 (2016), 487-494. 3, 4

[15] C. S. Ryoo, T. Kim, R. P. Agarwal, A numerical investigation of the roots of q-polynomials, Int. J. Comput. Math., 83 (2006), 223-234. 3

[16] Y. Simsek, Twisted (h, q)-Bernoulli numbers and polynomials related to twisted (h, q)-zeta function and L-function, J. Math. Anal. Appl., 324 (2006), 790-804.

[17] Y. Simsek, Generating functions of the twisted Bernoulli numbers and polynomials, Adv. Stud. Contemp. Math., 16 (2008), 251-278. 1, 1 